\title{
Relationship between Demographic Factors and Undergraduates' Cyberbullying Experiences in Public Universities in Malaysia
}

\section{David Obafemi Adebayo}

Department of Counsellor Education, Faculty of Education, University of Ilorin, Ilorin, Nigeria, adebayo.do@unilorin.edu.ng

\section{Mohd Tajudin Ninggal}

Department of Educational Foundations, School of Education, Faculty of Social Sciences and Humanities, Universiti Teknologi Malaysia, tajma28@utm.my

Foluke Nike Bolu-Steve

Department of Counsellor Education, Faculty of Education, University of Ilorin, Ilorin, Nigeria, folukebolu@gmail.com

In modern times, social media platforms have become veritable tools of communication and interaction amongst young adults in the university environment as well as the larger society. As important as it is, the platform has continued to cause serious psychological and emotional trauma to users through cyberbullying activities. This study examined the relationship between demographic factors and undergraduates' cyberbullying experiences in public universities in Malaysia. It was a quantitative study which adopted survey design. Self-designed questionnaire entitled Demographic factors and undergraduates' cyberbullying experiences was used for data collection. The instrument yielded coefficient of .92 using the Cronbach's alpha reliability. A total of 400 undergraduates were purposively selected at public university in west Malaysia, Johor. One research question was raised and three hypotheses were tested. Data collected were analysed using Structural Equation Modelling Partial Least Squares 3 (SEM-PLS3. The findings revealed that there was significant relationship between the demographic factors: gender and programme of study but no significance for race. Hence, gender and programme of study significantly predict cyberbullying experiences of undergraduates than racial affiliations. It was recommended that effective counselling be introduced in order to assist victims of cyber assaults overcome the trauma suffered, and also stem the wave of cyberbullying among undergraduates.

Keywords: demographic factors, undergraduates, cyberbullying, public universities, Malaysia

Citation: Adebayo, D. O., Ninggal, M. T., \& Bolu-Steve, F. N. (2020). Relationship between Demographic Factors and Undergraduates' Cyberbullying Experiences in Public Universities in Malaysia. International Journal of Instruction, 13(1), 901-914. https://doi.org/10.29333/iji.2020.13158a 


\section{INTRODUCTION}

In modern days of increasing technological advancements, the behaviour of bullying has changed its nature from one of physical, relational or verbal to the one that is perpetrated through the cyberspace. Thus, an important area of study requiring more of researchers' attention in these modern times is the increasing rate of technology-induced harassments, which include embarrassing social media experiences, inconvenient jesting through unhealthy pictorial or symbolic description of persons, mean text messages: resulting to serious emotional trauma, psychological torture, self-inflicted isolation due to group rejection, and even suicidal thoughts and performance of same; all as a result of deliberate attempts by young internet/social media users to use sophisticated information and communication technologies to cause harms, pains, and long-term injury to others, without physically meeting them (Keith and Martin, 2005; Campbell, 2012). This situation is no other than the menace called social media or electronic bullying, which in this study is regarded as cyberbullying.

The advent of social media platforms and smartphone devices have drastically reduced the human to human interactions, with the attendant effect that individuals have little or no empathy on other users of the platforms, while unwholesome behaviours are the norm in the present world (Adeboye, 2018). A growing and emerging problem that seriously affects university undergraduates and the youth in general, regardless of their gender, race, colour, personality or language (demography) across the world is cyberbullying. By demography, it implies the study of human populations and their respective changes over a period of time. In this study, the demographic components that were examined are gender, and race or ethnicity. In terms of gender, male and female undergraduates; whereas, under race/ethnicity, participants (undergraduates) were clustered into Malay, Chinese, as well as Indian and others. Cyberbullying affects the society at large, regardless of social status or class; and it can cause irreparable psychological damage to people, ranging from low self-esteem, depression, feelings of inadequacies, poor self-concept as well as suicide ideation. The broad term of bullying is seen as a global and widespread phenomenon occurring in different social contexts of schoolyard, workplace, and more recently, technology (cyber) (Fanti, Demetriou and Hawa, 2012; Abu-bakar, 2015).

In terms of susceptibility to cyberbullying attacks, Agatson et al., (2007) reported that male undergraduates seem to be less of victims of cyberbullying than female undergraduates. Female students are more cyberbullied than males; as a matter of fact, males are either bullies or bully-victims, while most females are largely reported as victims. This implies that female students are more of victims of social media bullying than their male counterparts (who mostly indulged in cyberbullying behaviours), particularly, university undergraduates (Agatson et al., (2007). In contrast, Balakrishnan (2015) concluded that due to the relative newness of social media bullying, the gender that is mostly affected by cyberbullying may not be easily determined because both gender appears equally affected in most cases. Many university students suffer in silence due to the adverse effects of online assaults suffered. While some can let go, many often resort to acts such as suicidal tendencies because of the psychological challenges 
experienced after the cyberbullying. Youngsters often feel ashamed, assume their world has crumbled, feel everyone has seen the online bullying, hence, the need to find solace in either hurting other students (becoming bully-victims), or isolating themselves from the society, including contemplating suicides (Nortal et al., 2013). Thus, the current study focuses on relationship between demographic factors and undergraduates cyberbullying experiences in Malaysian public universities.

\section{Problem Statement}

The advent of technology has continued to change the patterns of communication, interactions and relationships among young adults, particularly those in the university. Due to these various changes, undergraduates hardly make new physical friends, rather, new associates are continuously added on the cyberspace; and this has also changed the way and manner in which bullying is perpetrated in the university system. The social media platforms offer new ways by which bullying is perpetrated and this has continued to increase emotional and psychological trauma suffered by many youngsters regardless of gender, course of study and racial or ethnic group. Lu (2015) carried out a study on the influence of pathological internet use and demographic factors on pathological internet use among 1493 surveyed undergraduates; $1.2 \%$ prevalence of PIU was reported among undergraduates in a Malaysian public university; the author also found that undergraduates spent between 19 to 68 hours on the internet weekly, that is, at least about 6.5 hours on a daily basis; thus, the researcher found from the findings that majorly, undergraduates engaged in online games (MMORPG) and social networking sites (SNS), which were influenced by cognitive distortion, motivation via access to the internet, and stressful life events (boredom, depression) contributing 65\% variance of PIU (Pathological Internet Use). As it has been established that undergraduates spend a considerable number of hours on social media platforms on a daily basis, this study is therefore aimed at determining the relationship or link between motivations for social media use, demography, personality and cyberbullying behaviours among undergraduates studying in a Malaysian public university.

Ling, Ramadass, Altaher and Arjuman (2011) also identified 58 persons (29\%) at-risk of extreme internet use among 203 Malaysian undergraduates and the study asserted that individuals at ages 18 to 25 years were more vulnerable to the extreme use of the internet, called pathological internet use, particularly university or college students. This is because university students are considered as often having more free time to themselves, have easy access to the internet, as well as a more rational reason in the environment (Ling et al., 2011). Therefore, if individuals within the age bracket 18 to 25 years were found to be engaging in the extreme use of the internet, particularly for nonacademic purposes, there is the need to establish if the frequency of use of social media by undergraduates has any relationship or link with cyberbullying behaviours; as well as the motivations for such frequent visits to social media platforms, and personality traits of users.

The Malaysia Crime Prevention Foundation in April, 2017 gave a detailed analysis of cyberbullying behaviours perpetrated by university and other categories of students, from the statistics obtained from Cyber-Security Malaysia's first quarter report of 2017 
in which it was revealed that there were (300) cyber harassment cases in 2012, (512) 2013, 2014 (550), 2015 (442) and 2016 (529); a total of 2333 general cases of cyberbullying. However, according to the report, cyberbullying among students of tertiary institutions is considered more serious, with 250 cases reported in 2012, (389) 2013, 2014 (291), 2015 (256) and 2016 (338). In all, a total of 1524 (65\% of 2333) cases of cyberbullying have been reported and recorded among university and high school students in the past six years; this is not including the variously unreported cases because of the inherent shame and ridicule that victims are most likely to face should they report the attacks (Malaysia Crime Prevention Foundation, 2017). Hence, the reason for this study to determine the relationship between demographic factors and students' cyberbullying experiences in public universities, particularly in west Malaysia.

\section{Objective of the Study}

The objective of this study is to determine the relationship between demographic factors and undergraduates' cyberbullying experiences in Malaysian public universities. Specifically, it is to examine the correlations of demographic variables of gender, race/ethnicity, programme (course) of study and cyberbullying experiences of youngsters in public universities in west Malaysia.

\section{Research Question}

What is the relationship between demographic factors and undergraduates' cyberbullying experiences in Malaysian public universities?

\section{Research Hypotheses}

The following research hypotheses were generated in the null form and tested in this study:

1. There is no significant relationship between demographic factors and undergraduates' cyberbullying experiences in Malaysian public universities based on gender.

2. There is no significant relationship between demographic factors and undergraduates' cyberbullying experiences in Malaysian public universities based on race.

There is no significant relationship between demographic factors and undergraduates' cyberbullying experiences in Malaysian public universities based on programme of study.

\section{METHOD}

This is a quantitative study and it adopted survey design. Survey was deemed more suitable and appropriate because of the nature of the study; i.e., one dealing with large population from which samples were drawn for the study. The population comprised all undergraduates in the selected public university in west Malaysia. A total of 400 participants were purposively selected from the three faculties that were selected through a process of cluster sampling technique. 
Purposive sampling was adopted in order to ensure that all the races represented were fairly enlisted to participate in the study. A self-designed questionnaire was used for data collection. The questionnaire was entitled "Engagement on Social Media and Cyberbullying Behaviours (ESMU \& CBB Questionnaire)". The researchers decided to design the questionnaire in order to be able to give careful consideration to the cultural and religious practices of the respondents in this part of the country without any form of bias. The questionnaire was divided into two parts (A \& B). Part A focused on respondents' demographic details, while part B consisted of items on demographic factors, social media use and cyberbullying among undergraduates. The instrument was tailored towards a Four-point Likert type format on a continuum of 4-1 scoring. The instrument was subjected to a Cronbach's alpha method of reliability, and it yielded a coefficient of 0.92 , thus, making it reliable for use as the data gathering tool for this study. Also, the SEM-PLS carried out its own validity and reliability before the results were run; this further confirmed the usability of the instrument and its results. Participation was voluntary, thus, respondents offered to actively participate in the study Data collected were analysed using the descriptive statistical tools and SEM-PLS3 While the demographic aspects were analysed with the use of percentage, mean and standard deviation, the null hypotheses were tested with the SEM-PLS3. All hypotheses were tested at 0.05 alpha level of significance.

\section{FINDINGS}

\section{Demographic Data}

The study was conducted among 400 university undergraduates from three faculties. In this section of the study, the demographic profile of respondents which is referred to as the description of the attribute of the samples were presented on their individual characteristics based on gender, frequency of daily use of social media platforms, programme of study, race/ethnicity, and whether or not they have experienced cyberbullying assaults before. The section below elaborates on the results obtained.

\section{Gender}

For gender, the results indicated that one hundred and fifty-two (152), 38.0\% of the respondents were males and two hundred and forty-eight (248), 62.0\% of the respondents were females. This implies that more female undergraduates participated in the study than their male counterparts. It also suggests that there are more female undergraduates than there are male undergraduates in the selected university. Figure 1 presents the result. 


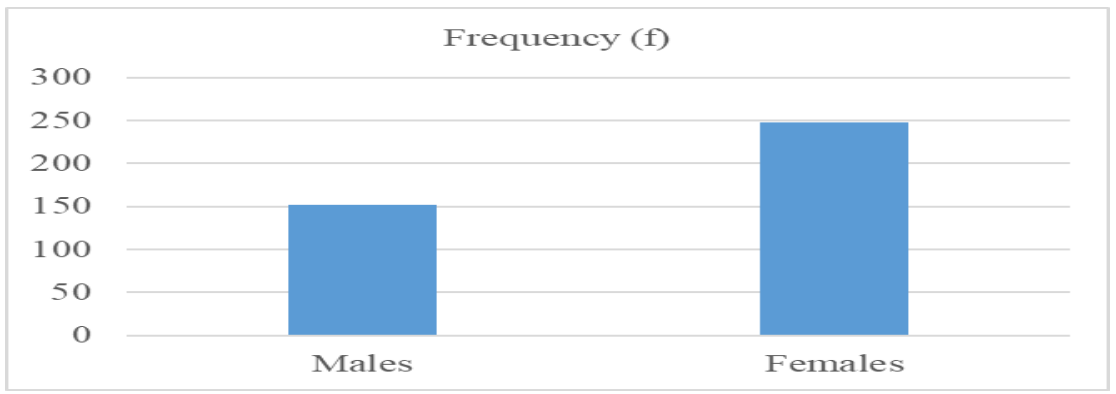

Figure 1

Gender Distribution of Respondents

\section{Frequency of Daily Use of Social Media Platforms}

The daily frequency of the respondents' usage of social media platforms was grouped into less than 1 hour, 1 to 3 hours, 4 to 6 hours and above 6 hours. The result indicates that thirteen (13), $3.3 \%$ of the respondents spend less than an hour daily on the social media, one hundred and six (106), 26.5\% spend 1 to 3 hours, one hundred and thirtyeight (138), $34.5 \%$ of the respondents spend between 4 to 6 hours and one hundred and forty-three (143), 35.8\% spend above 6 hours daily on social media platforms. This implies that undergraduates spent more than 6 hours daily on social media platforms.

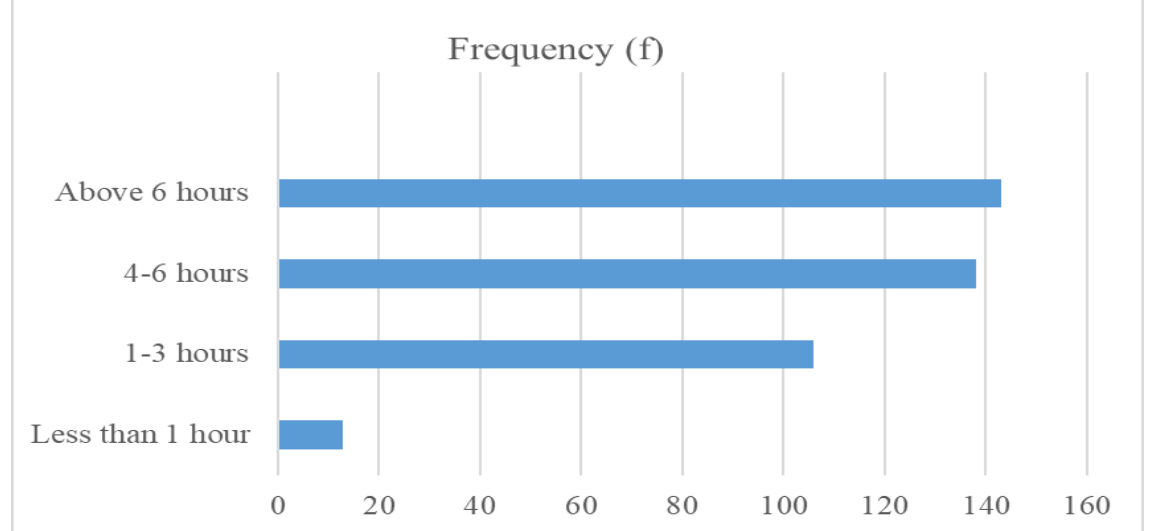

Figure 2

Respondents' Distribution on Daily Frequency of Usage of Social Media Platforms

\section{Race/Ethnicity}

The race/ethnicity was categorized into four: Malays, Chinese, Indians and Others. The analysis revealed that two hundred and eighteen (218), 54.5\% of the respondents were Malays, while eighty-seven (87), $21.8 \%$ were Chinese, whereas, there were twentyseven (27), 6.8\% Indians and sixty-eight (68) $17.0 \%$ other races/ethnic nationals that participated in the study. 


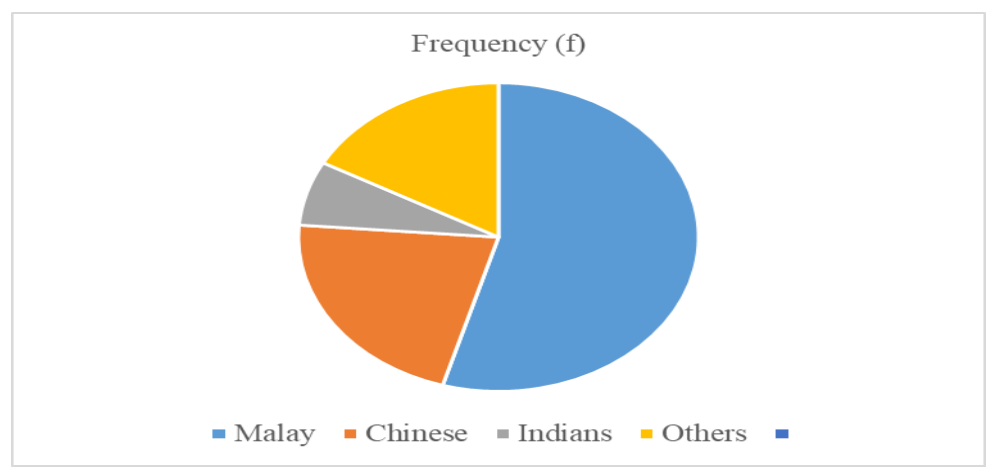

Figure 3

Respondents' Race/Ethnic Distribution

\section{Programme of Study}

According to the results, $25.0 \%(\mathrm{~N}=100)$ consisted of computing undergraduates, $37.5 \%$ $(\mathrm{N}=150)$ electrical engineering students, and $37.5 \%(\mathrm{~N}=150)$ undergraduates in various management programmes participated in the study. From the result it can be deduced that there are more undergraduates in both electrical engineering and management than computing.

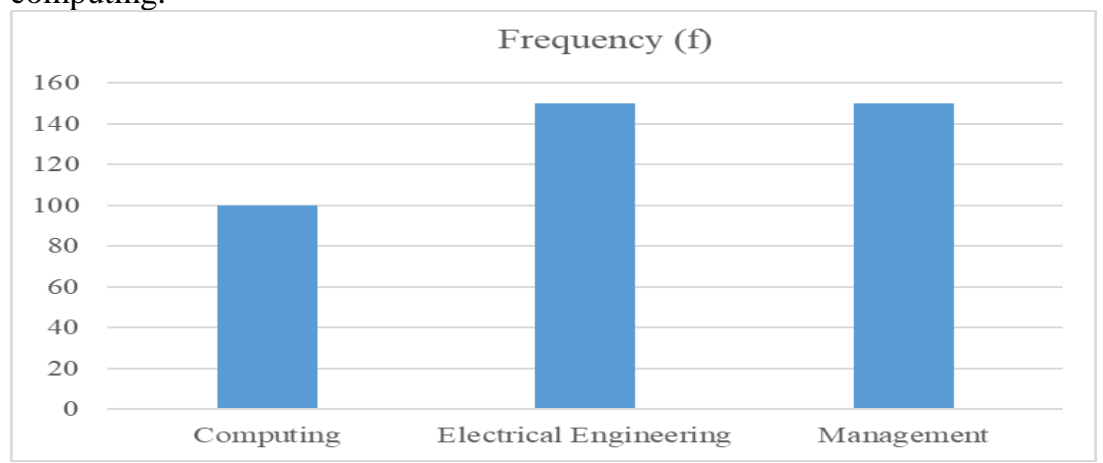

Figure 4

Respondents' Programme of Study

\section{Experience of Cyberbullying}

The distribution of the respondents based on their cyberbullying experiences indicated that two hundred and forty-four (244) $(61 \%)$ of the respondents have experienced cyberbullying, while 156 (39\%) have never experienced cyberbullying. This further confirms that cyberbullying behaviour is rampant among university undergraduates. 


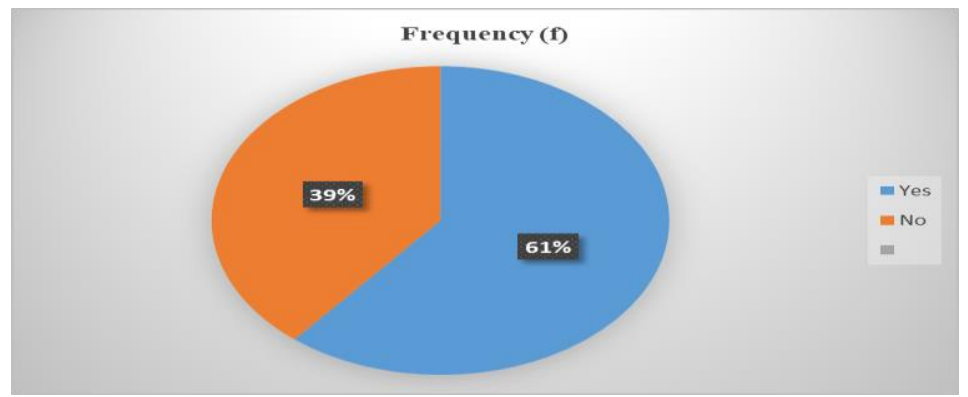

Figure 5

Respondents' Experience of Cyberbullying

Hypothesis 1: There is no significant relationship between demographic factors and undergraduates' cyberbullying experiences in Malaysian public universities based on gender.

\section{Direct Relationship of Gender and Cyberbullying Behaviors}

Hypothesis one focused on identifying the relationship that undergraduates' gender has on cyberbullying behaviours (CBB). The path analysis was used in order to achieve this hypothesis as shown below in figure 6

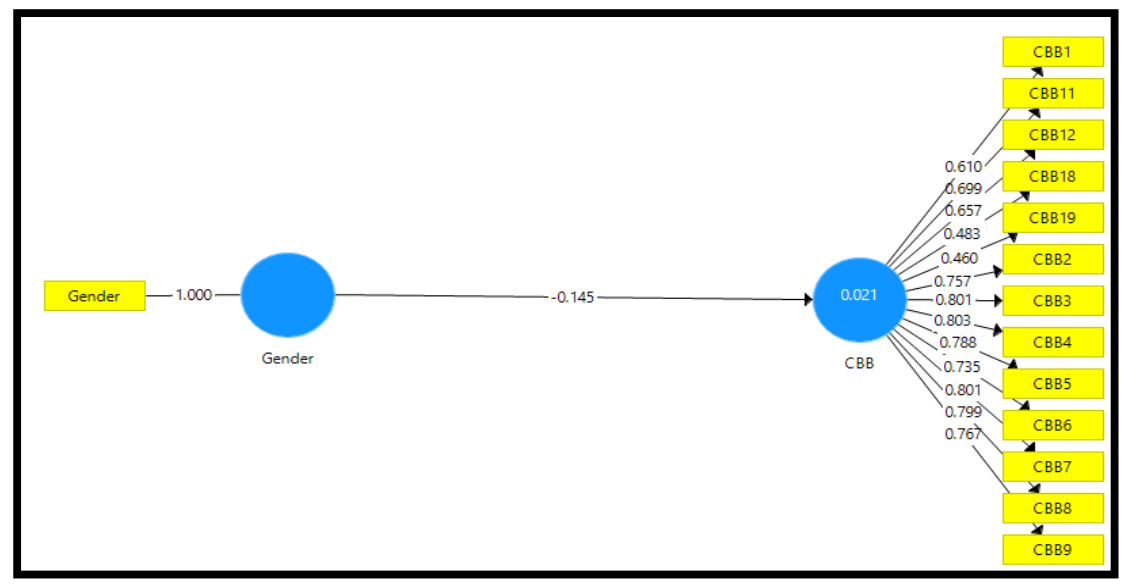

Figure 6

Gender and Cyberbullying Behaviours

Table 1

T-statistics and P-value of Gender on Cyberbullying Behaviours

\begin{tabular}{lllccl}
\hline & Original Sample $(\mathrm{O})$ & Mean(M) & SD & T Statistics & P Values \\
\hline Gender-> CBB & -0.145 & -0.166 & 0.042 & 3.459 & 0.001 \\
\hline
\end{tabular}

*Significant; $\mathrm{p}<0.05$ 
The result of the path analysis shows that gender $(\mathrm{G})$ has a significant direct negative relationship with cyberbullying behaviours of undergraduates (CBB). The relationship is negative with a path coefficient $(\beta=-0.145, \mathrm{t} \geq \pm 1.96)$. The standard decision rule of Byrne (2010) (t-value greater than or equal to 1.96 and $p$-value is $\leq 0.05$ ) is also applicable in this hypothesis in order to determine the significances of the path coefficient between the dependent variable and the independent variables. Therefore, since the t-value is $\geq \pm 1.96$, it thus implies that the relationship between gender and cyberbullying is significant, and gender has an influence on undergraduates' cyberbullying behaviours. A continuous increase in the population of female undergraduates means further decline in cyberbullying behaviours, whereas an increase in the number of male students implies more experiences of cyberbullying because most male undergraduates reported not to have experienced cyberbullying, which means females are more of the victims than males who appear mostly as the perpetrators of the act of cyberbullying. Thus, the null hypothesis which states that there is no significant relationship between gender of undergraduates and cyberbullying behaviours is not accepted. In this study gender accounts for $.021 \%$ on cyberbullying behaviours.

Hypothesis 2: There is no significant relationship between demographic factors and undergraduates' cyberbullying experiences in Malaysian public universities based on race.

\section{Direct Relationship of Race/Ethnicity and Cyberbullying Behaviours}

Hypothesis two focused on identifying the relationship that undergraduates' race/ethnicity $(\mathrm{RE})$ has on their cyberbullying behaviours $(\mathrm{CBB})$. The path analysis was used in order to test this null hypothesis. Figure 7 shows the relationship between the two variables.

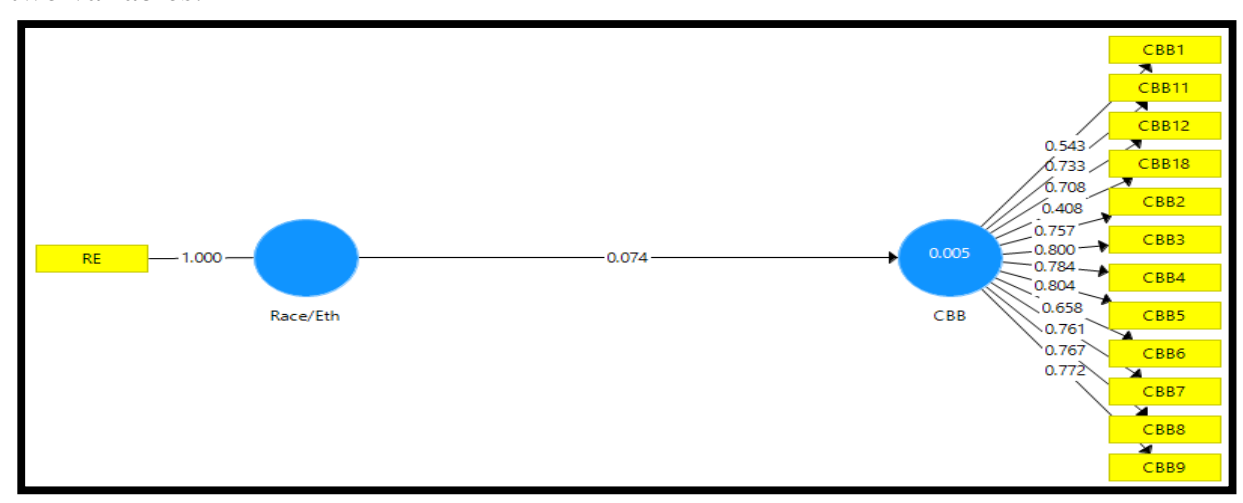

Figure 7

Race/Ethnicity and Cyberbullying Behaviours 
Table 2

T-statistics and P-value of Race/Ethnicity on Cyberbullying Behaviours

\begin{tabular}{llllll}
\hline & $\begin{array}{l}\text { Original } \\
\text { Sample } \\
(\mathrm{O})\end{array}$ & Mean $(\mathrm{M})$ & SD & T Statistics & P Values \\
\hline RE-> & 0.074 & 0.081 & 0.116 & 0.639 & 0.523 \\
CBB & & & & & \\
\hline
\end{tabular}

*Significant; $\mathrm{p}>0.05$

Based on the path analysis of figure 7 on undergraduates' race/ethnicity on their cyberbullying behaviours, the result shows that there is a positive but not significant relationship. The relationship is positive but not significant with path coefficient $(\beta=$ $0.074, \mathrm{t} \leq \pm 1.96$ ). Based on the t-statistics of .639 and $\mathrm{p}$-value of .523 , it means race/ethnicity has no influence on cyberbullying behaviours. Only $.005 \%$ of race/ethnicity was accounted for in cyberbullying behaviours. Thus, the null hypothesis which states that there is no significant relationship between undergraduates' race/ethnicity and cyberbullying behaviours was accepted.

Hypothesis 3: There is no significant relationship between demographic factors and undergraduates' cyberbullying experiences in Malaysian public universities based on programme of study.

\section{Direct Relationship of Programme of Study and Cyberbullying Behaviours}

Hypothesis three focused on identifying the relationship that undergraduates' programme of study (PS) has on cyberbullying behaviours (CBB). Path analysis was used in order to test the null hypothesis. Figure 8 shows the relationship between the two variables.

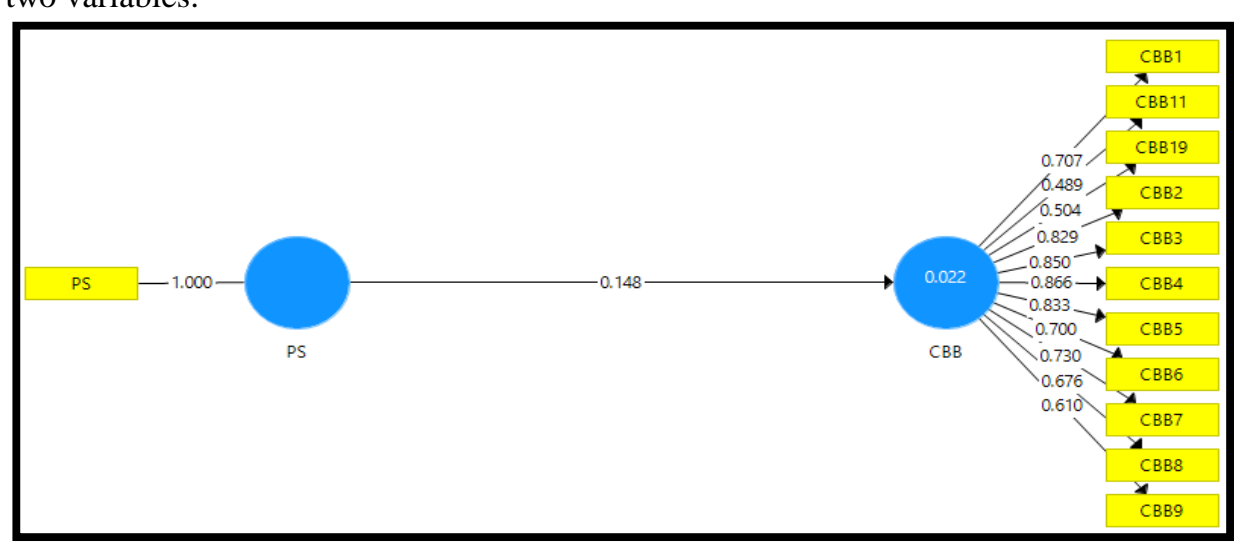

Figure 8

Programme of Study and Cyberbullying Behaviours 
Table 3

T-statistics and P-value of Programme of Study on Cyberbullying Behaviours

\begin{tabular}{llllll}
\hline & $\begin{array}{l}\text { Original } \\
\text { Sample } \\
(\mathrm{O})\end{array}$ & Mean(M) & SD & T Statistics & P Values \\
\hline PS-> CBB & 0.148 & 0.171 & 0.064 & 2.328 & 0.020 \\
\hline
\end{tabular}

*Significant; $\mathrm{p}<0.05$

The result of the path analysis shows that programme of study (PS) has a significant and direct relationship with undergraduates' cyberbullying behaviours (CBB). The relationship is positive with a path coefficient $(\beta=0.148, \mathrm{t} \geq \pm 1.96)$. According to Byrne (2010), the standard decision rule for a relationship between constructs to be significant is ( $\mathrm{t}$-value greater than or equal to 1.96 and $\mathrm{p}$-value is $\leq 0.05$ ). This is used here to decide the significance of the path coefficient between the dependent variable and the independent variable. Therefore, since the t-value is $\geq \pm 1.96$, it therefore, means the relationship between programme of study and cyberbullying behaviour is significant and that programme of study has an influence on undergraduates' cyberbullying behaviours. Thus, the null hypothesis which states that there is no significant relationship between programme of study and cyberbullying behaviour is not accepted. In this study, the programme of study accounts for $.022 \%$ on cyberbullying behaviours.

\section{DISCUSSION AND CONCLUSION}

The findings of this study showed that a significant relationship exists between gender of undergraduates and cyberbullying behaviours, though it is an inverse (negative) relationship. It thus implies that if a particular gender (say females) increases, cyberbullying behaviours tend to reduce, and if the other gender (males) declines, cyberbullying experiences of victims reduces while increased male enrolments imply an increase in cyberbullying experiences by female undergraduates. This is consistent with the study of Wright et al., (2015) in which the researchers affirm that Chinese and Japanese females were more of victims of cyberbullying than Chinese and Japanese males who were in the majority of cyberbullies.

This finding also corroborates the study of Agatson et al., (2007) which reported that male undergraduates seem to be less of victims of cyberbullying experiences than female undergraduates. Female students are more cyberbullied than males; as a matter of fact, males are either bullies or bully-victims, while most females are largely reported as victims of cyber harassments. Also, the finding confirms that of $\mathrm{Li}$ (2006) which found that male students of high schools were more cyberbullies than female students, the majority of whom were victims of cyberbullying assaults. This study however sharply contrasts with the findings of Balakrishnan (2015) which concluded that due to the relative newness of social media bullying, the gender that is mostly affected by cyberbullying may not be easily determined because both genders appears equally affected in most cases. 
The results further showed that there is no significant relationship between race/ethnicity and cyberbullying behaviours. This implies that the behaviour of cyberbullying is an individual thing, and not one of a racial or ethnic colouration. This supports Glasser's (2000) Choice theory that opines that each human being is the author of his or her behaviour and not as a result of an unconscious or invisible element. This finding however sharply contrasts to that of Chan and Wong (2015) who found that culture was a strong predictor of cyberbullying behaviours; hence, the authors concluded that cultural differences between the west and general Chinese culture may have given rise to the differences in terms of prevalence, nature, severity, and features of cyberbullying. The conclusion of the authors further lends credence to the earlier findings of Li (2007) and $\mathrm{Li}$ (2008) which also confirmed that culture predicts cyberbullying behaviours and its perpetration was higher in Chinese societies. These may further provide support to the findings of this study from the cross-tabulation which revealed that there were more Chinese undergraduates in electrical engineering than they are in management and computing. Furthermore, from the cross tabulation results obtained, it showed that undergraduates in electrical engineering were less frequent users of social media platforms than their colleagues in faculties of computing and management; it also revealed that electrical engineering students were less of victims of cyberbullying behaviours than computing and management students. The reason for this may be because of the excess workloads of electrical engineering students, which does not afford them the opportunity for social media activities, which ultimately gives them positive hedges over others as evidenced in their academic records published by the university during convocation programmes. However, it may also have been why Li (2008) concluded that if the increasingly higher percentage of cyberbullying behaviours amongst Chinese students was not checked, it could become a serious global problem. Furthermore, it is interesting to note that cyberbullies may not necessarily be frequent users of social media platforms. The only group that gets harassed are the victims who spend a considerable number of hours online. This is also corroborative of the study of Notar et al., (2013) asserting that cyberbullies may not necessarily require to spend long hours on social media as long as the raw materials required for bullying their victims (victims' online profiles) are within their (cyberbullies) reach.

Findings also revealed that there is a significant relationship between programme of study and cyberbullying behaviours. This was also revealed in the cross-tabulation analysis which indicated that undergraduates of management spent more time on social media platforms, and also topped the list of victims of cyberbullying experiences, while computing and electrical engineering students came next. Undergraduates of electrical engineering were less of victims of cyberbullying and majority of them were males, unlike management and computing that had more female undergraduates. Previous researchers have only concentrated on defining what cyberbullying is and its nature across different schools, culture, colour, and its nature, with none seeming to have considered the programme or course of study of the university students who had been cyberbullying victims or cyberbullies. This is supportive of Wright's et al., (2015) view that though cyber-aggression is on the increase, researches on it have been slower in most Asian countries. Thus, this gap has been succinctly filled by the present study. 
Hence, there is a significant relationship between undergraduates' programmes of study and cyberbullying behaviours. This corroborates the findings of Eksi (2012) that young people in the universities have in recent times sought helps from counselling practitioners and mental health experts due to their incessant experiences of cyber assaults.

Based on the findings of this study, it was concluded that; the behaviour of cyberbullying has no ethnic or racial colouration or tone, rather, it is an individual behaviour, just like no two individuals are exactly the same in terms of their personality. It established that being a Malay, Chinese, Indian or others has nothing to do with cyberbullying behaviour, as anyone from any race or ethnic group can be a potential cyberbully. It was also revealed that there were more Chinese undergraduates in electrical engineering than they are in management and computing.

\section{RECOMMENDATIONS}

The following suggestions are recommended based on the findings of this study:

a. Academic motivations for social media use should be separated from social motives in order to avoid undue interferences in the results obtained. This becomes imperative so as to avoid misinterpretation of research findings.

b. A similar study is recommended for other universities in Malaysia in order to adequately extend literature in this regard and to ultimately determine the prevalence of cyberbullying among undergraduates in other Malaysian universities, particularly, the public universities.

Age factor may have also contributed to undergraduates' frequent use of social media platforms. Future studies can focus on graduate students' use of social media in order to ascertain the frequency of use and cyberbullying amongst postgraduate students as established for undergraduates in this study.

\section{REFERENCES}

Abu Bakar, H.S. (2015). Emergence themes of cyberbullying among adolescents in Malaysia. International Journal of Adolescence and Youth, 20(4), 393-406.

Adeboye, E.A (2018). Good neighbourliness II: Open Heavens Daily Devotional (May 26, 2018) Module 2018. Lagos: Publication of Christ the Redeemers' Ministry (CRM).

Balakrishnan, V. (2015). Cyberbullying among young adults in Malaysia: The roles of gender, age and Internet frequency. Computers in Human Behaviour, 46, 149-157.

Brown, J. D. (1996). Testing in language programs. Upper Saddle River, NJ: Prentice Hall Regents.

Campbell, M.A. (2012). Cyber bullying: An old problem in a new guise? Journal of Psychologists and Counsellors in Schools, 22(1), https://doi.org/10.1017/jgc.2012.10. 
Campbell, M. A., Slee, P. T., Spears, B., Buffer, D., \& Kift, S. (2013). Do cyberbullies suffer too? Cyberbullies' perception of the harm they cause others and to their own mental health. School Psychology International, 34(6), 613-629.

Cassidy, W., Jackson, M., \& Brown, K.N. (2009). Sticks and stones can break my bones, but how can pixels hurt me? Students' experiences with cyberbullying. School Psychology International, 30(4), 383-402. doi:10.1177/0143034309106948.

Chan, H. C. O., \& Wong, D. S. W. (2015). Traditional school bullying and cyberbullying in Chinese societies: Prevalence and a review of the whole-school intervention approach. Aggression and Violent Behavior, 23, 98-108.

Cross, D., Barnes, A., Papageorgiou, A., Hadwen, K., Hearn, L., \& Lester, L. (2015). A social-ecological framework for understanding and reducing cyberbullying behaviours. Aggression and Violent Behaviour, 23, 109-117.

Eksi, F. (2012). Examination of narcissistic personality traits' predicting level of internet addiction and cyber Bullying through path analysis. Journal of Educational Sciences, Theory and Practice, 12(3), 1694-1706.

Fanti, K. A., Demetriou, A. G., \& Hawa, V. V. (2012). Cyberbullying: Development, consequences, risk and protective factors-A longitudinal study of cyberbullying: Examining risk and protective factors. European Journal of Developmental Psychology, 9(2), 168-181.

Hair, J. F., Hult, G. T. M., Ringle, C. M., \& Sarstedt, M. (2014). A primer on partial least squares structural equation modelling (PLS-SEM). LA: Sage Publications.

Li, Q. (2007). New bottle but old wine: A research of cyberbullying in schools. Computers in Human Behaviour, 23(4), 1777-1791.

$\mathrm{Li}$, Q. (2008). Cross-cultural comparison of adolescents' experience related to cyberbullying. Educational Research, 50(3), 223-234.

Malaysia Crime Prevention Foundation (2017). On the alert for cyberbullying. CyberSecurity Malaysia Report of April, 2017.

Nortar, C.E., Padgett, S., \& Roden, J. (2013). Cyberbullying: A review of the literature. Universal Journal of Educational Research, l(1), 1-9. doi: 10.13189/ujer.2013.010101.

Wright, M. F., Aoyama, I., Kamble, S. V., Li, Z., Soudi, .S., Lei, L., \& Shu, C. (2015). Peer attachment and cyber aggression involvement among Chinese, Indian, and Japanese adolescents. Societies, 5(2), 339-353. doi:10.3390/soc5020339. 